\title{
Yet-Ming Chiang to give plenary address at 2011 MRS Spring Meeting
}

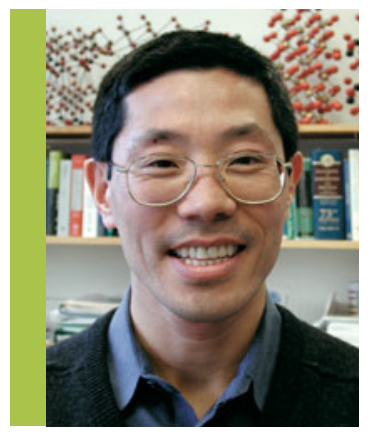

$\mathbf{Y}^{\mathrm{e}}$ et-Ming Chiang, a co-founder of A123 Systems, will give the plenary address at the 2011 Materials Research Society Spring Meeting in San Francisco. The plenary session will be held Wednesday, April 27th, at 6:30 p.m. in the San Francisco Marriott Marquis.
Chiang is also the Kyocera Professor of Ceramics in the Materials Science and Engineering Department at the Massachusetts Institute of Technology, where he focuses on the design, synthesis, and characterization of advanced inorganic materials and related devices. His current research interests include new cathode and anode materials for lithium ion batteries, phase transformations in electroactive materials, electrochemical device design, electrochemical-tomechanical energy conversion, self-assembling colloids, and MRS the stability and defect chemical properties of interfaces in inorganic materials.

In 1987, Chiang co-founded American Superconductor. He has published widely in materials science and engineering, and served on numerous government panels and editorial boards of journals in his field. Chiang received an SB degree in materials science and engineering in 1980 and an ScD degree in ceramics in 1985, both from MIT.

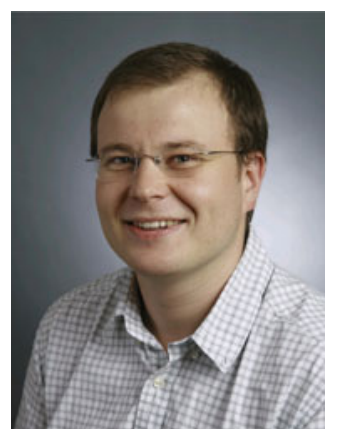

\section{Dmitri Talapin named 2011 MRS Outstanding Young Investigator for inorganic nanocrystals}

D mitri V. Talapin, assistant professor in the Department of Chemistry at the University of Chicago, has been named the 2011 Materials Research Society Outstanding Young Investigator. Talapin was cited for "methodological developments of synthesis and selfassembly of inorganic nanocrystals and for fundamental studies transforming colloidal nanostructures into electronic and optoelectronic materials." He will deliver an award talk at the Materials Research Society Spring Meeting in San Francisco.

Talapin has provided key advances toward three of the overarching goals of the field of nanomaterials. He contributed significantly to the methodology of synthesis of nanostructures with complex shapes and compositions. A key development in this area is his discovery and physical/theoretical characterization of nano-heterostructures of "mixed dimensionality" - semiconducting materials with different confinement regimes for electrons and holes. In parallel, Talapin synthesized the first SnTe quantum dots and investigated their optical and electronic properties. He also made key contributions to elucidating the fundamental aspects of self-assembly phenomena, which is critical to rationally creating and controlling new nanostructures.

Talapin has made first field-effect devices assembled of nanocrystals and advanced scientific understanding of the collective interactions in nanocrystal superlattices, which ultimately will establish the fundamental bases for device applications of these materials. And most recently, Talapin found that metal chalcogenide complexes can serve as ligands for colloidal nanocrystals and nanowires and can be thermally converted to semiconducting phases to generate all-inorganic highly conductive nanocrystal solids. Using this approach, he has demonstrated that arrays of semiconductor nanocrystals can develop high charge carrier mobility, suitable for designing efficient field-effect transistors, photodetectors, and solar cells.

Talapin received his diploma in chemistry from Belarusian State University in 1996 and his $\mathrm{PhD}$ degree in chemistry from the University of Hamburg in 2002. After serving as a postdoctoral fellow at the IBM T.J. Watson Research Center (2003-2005) and a staff scientist at the Molecular Foundry in the Lawrence Berkeley National Laboratory (2005-2007), he joined the faculty at the University of Chicago in 2007. In 2010, he received the

Camille Dreyfus Teacher-Scholar Award. Among Talapin's other honors are the David and Lucile Packard Fellowship (2009), Sloan Fellowship (2009), NSF CAREER Award (2008), LMUexcellent Fellowship, Germany (2007), IBM Invention Achievement Award (2004, 2006), Belarus National Academy of Sciences Award (1995); and 1st Prize of the USSR Chemistry Olympiad (1991). Talapin has eight patents and over 100 publications. He is an editorial board member of Nanoscale, published by the Royal Society of Chemistry and an invited editor for a special issue of the Journal of Materials Chemistry. 